\title{
Relación fenética de especies del orden Poales de interés económico en la laguna El Paraíso, Huacho, 2017
}

\author{
Phenetic relationship of Poales species of economic interest in the El Paraíso lagoon, \\ Huacho, 2017
}

Hermila B. Díaz Pillasca', Zoila Honorio Durand², Carmen Rojas Zenozain', Miguel A. Durand Meza

\section{RESUMEN}

Objetivo: Estimar la relación fenética de las especies del orden Poales de interés económico de la laguna El Paraíso, Huacho - 2017. Métodos: Para lo cual se aplicó el diseño de una sola casilla; donde la población estuvo constituida por todos los individuos de las especies seleccionadas; de la cual se extrajo una muestra de 43 individuos, mediante un diseño aleatorio estratificado; eligiéndose 34 caracteres cualitativos y 13 cuantitativos; utilizados para estimar variabilidad y distancia fenética dentro y entre especies, mediante estadística descriptiva, inferencial (ANVA y Tukey) y Taxonomía Numérica, a través del programa Past 3.0. Resultados: Los caracteres cuantitativos de interés económico (longitud de culmo) muestran una baja variabilidad fenotípica (CV < 12\%) intra e interespecífica, presumiblemente por influencia ambiental; la distancia fenética intraespecífica es estrecha en las tres especies evaluadas, siendo T. dominguensis, la que muestra mayor número de formas fenotípicas; $y, C$. laevigatus tiene una mayor distancia fenética con respecto a $S$. californicus y $T$. dominguensis; las mismas que son iguales entre sí. Conclusiones: El humedad es una comunidad compleja pero poco estable.

Palabras clave: Relación fenética, Poales, Interés económico.

\section{ABSTRACT}

Objective: Estimate the phenetic relationship of the Poales species of economic interest of the El Paraíso lagoon, Huacho - 2017. Methods: for which the design of a single box was applied; where the population was constituted by all the individuals of the selected species; from which a sample of 43 individuals was extracted, by means of a stratified random design; choosing 34 qualitative and 13 quantitative characters; used to estimate variability and phenetic distance within and between species, through descriptive, inferential statistics (ANVA and Tukey) and Numerical Taxonomy, through the Past 3.0. Results: The quantitative characters of economic interest (culm length) show a low phenotypic variability $(\mathrm{CV}<12 \%)$ intra and interspecific, presumably due to environmental influence; the intraspecific phenetic distance is narrow in the three evaluated species, being $\mathrm{T}$. dominguensis, the one that shows the greatest number of phenotypic forms; and, C. laevigatus has a greater phenetic distance with respect to $\mathrm{S}$. californicus and T. dominguensis; the same ones that are equal to each other. Conclusions: Humidity is a complex community but not very stable.

Keywords: Phenetic relationship, Poales, Economic interest.

\footnotetext{
Facultad de Ciencias. Universidad Nacional José Faustino Sánchez Carrión. Huacho, Perú.

${ }^{2}$ Facultad de Bromatología y Nutrición. Universidad Nacional José Faustino Sánchez Carrión. Huacho, Perú.
} 


\section{INTRODUCCIÓN}

Los humedales son ecosistemas de gran productividad; (Mitsch, 1993) por lo que se propugna su conservación y uso racional (Ramsar, 2016). Su importancia ha sido reiteradamente indicada por los bienes y servicios ambientales que brinda (Dugan, 1992). Por ello, los estudios para la conservación y uso racional de los humedales son numerosos; tanto a nivel mundial, latinoamericano y nacional (Mitsch, 1993; Moschella, 2012; Ramsar, 2016; Dugan, 1992; Aponte y Cano, 2013).

Más recientemente se ha comenzado a estudiar el rol de los humedales, como importante banco de germoplasma vegetal (Castro Gómez et al. 2015); que requiere, previamente, la caracterización fenotípica de la especie (Huaringa, Huanuqueño, Camarena y Chura 2013); lo que se logra con métodos estadísticos y taxonomía numérica (Núñez y Escobedo 2011; Crisci y Armengol, 1983).

En Perú, de la diversidad florística reportada para humedales costeros (Aponte \& Cano, 2013), destacan especies del género Typha y Schoenoplectus; al punto que humedales de Barranca, Huaura y Huaral han sido declarados Patrimonio Cultural de la Nación (Res. Viceministerial $N^{\circ}$ 054-2015-VMPCIC, 2015). De ellos, resalta la laguna "EI Paraíso", donde se explotan especies de los géneros arriba mencionados; y no estudiadas con fines de manejo y fitomejoramiento; vacío que se pretende cubrir, progresivamente, iniciándolo con la estimación fenética, bajo la hipótesis de que existe una relación fenética gradual dentro y entre las especies del orden Poales de interés económico en la laguna El Paraíso de Huacho, 2017.

Para lo cual se debe determinar las especies de importancia económica, evaluar la variabilidad intra e interespecífica, determinar la distancia fenética e identificar los principales caracteres cuantitativos que permitan selección con fines de fitomejoramiento.

La variabilidad fenotípica dentro y entre especies, implica la interacción genotipoambiente, y es el punto de partida para estudios genómicos y transcriptómicos; además, los humedales brindan a la sociedad bienes y servicios de uso directo e indirecto. Por todo ello, la estimación de la relación fenética de especies del orden Poales de interés económico en la laguna EI Paraíso de Huacho, se justifica plenamente por la trascendencia que tienen los humedales desde el punto de vista ecológico, científico, económico, turístico y paisajístico.

\section{MATERIALY MÉTODOS}

Este trabajo es descriptivo - transversal; con diseño de una sola casilla; donde la población tiende al infinito, al estar constituida por todas las repeticiones de evaluación de la unidad muestral; representada por cada individuo perteneciente a alguna de las especies consideradas en la evaluación. La muestra fue seleccionada mediante un diseño aleatorio estratificado con afijación simple; estimándose el tamaño de muestra mediante la fórmula para población infinita y variable categórica (Ostle, 1981) $: n=Z^{2} P . Q /(E E)^{2}$. Dónde: $Z=1,96\left(\alpha_{12}=0,05\right) ; P=0,99 ; Q=$ 0,$01 ; E E=0,1 ; n=39$.

La variable en estudio fue Relación fenética de los organismos, en base a 34 caracteres cualitativos y 13 cuantitativos; clasificados en las dimensiones doble estado (indicadores: presente/ausente y excluyentes) y multiestado; siendo sus indicadores cualitativos sin o con secuencia lógica y cuantitativos discretos y continuos.

El procedimiento para la obtención de datos (Crisci y Armengol, 1983), se inició con la selección de las especies, que forman las Unidades Taxonómicas Operativas (OTUs). Luego, se eligieron, aleatoriamente, el mismo número de individuos para cada especie (unidades intraespecíficas), de acuerdo al tamaño de muestra; pero se evaluaron 3 unidades más para Typha dominguensis. Se 
eligieron 47 caracteres; considerando el estado del carácter y su codificación; ello permitió construir la matriz básica de datos (MBD): los mismos que fueron introducidos en el programa Past 3.0, el cual internamente construye la matriz de distancias (MD) y aplicando la técnica de agrupación de la media aritmética no ponderada (UPGMA), elabora el fenograma correspondiente.

La variabilidad de cada unidad taxonómica (intra e interespecífica) se estimó mediante medidas de tendencia central y dispersión, en función de los caracteres cuali y cuantitativos; probándose diferencias significativas entre OTUs, mediante el análisis de varianza unifactorial y prueba de Tukey, a un nivel de confianza del $95 \%$.

\section{RESULTADOS}

La ubicación taxonómica de las especies seleccionadas y los resultados de los análisis estadísticos y fenéticos, se presentan en las tablas y figura siguientes.

En la Tabla 2, se presenta la estadística descriptiva de cada uno de los individuos de las especies evaluadas, considerando los 34 caracteres cualitativos. En ella se observa homogeneidad dentro y entre especies para la mediana $(M n=1)$; mientras que el $\mathrm{CV}$ indica heterogeneidad; donde Cyperus laevigatus tiene un valor de $\mathrm{CV}=51,08$ para cada individuo; en Typha dominguensis tienen un $\mathrm{CV}=37,70$; y los $\mathrm{CV}$ en individuos de Schoenoplectus californicus, no son todos iguales. Los valores de media y medidas de dispersión, en base a 13 caracteres cuantitativos; indican que existe variabilidad intrapoblacional; con una desviación estándar (S) mayor de 30 en todos los casos; y un CV cercano o superior al $200 \%$. Los promedios más bajos corresponden a individuos de C. laevigatus; mientras que los más altos pertenecen a los individuos de $T$. dominguensis.

La variabilidad de los 13 caracteres cuantitativos, en base al número de individuos evaluados para cada especie; por interés económico, se resalta que los valores más elevados para longitud del culmo y longitud del limbo corresponden a T. dominguensis, y los más bajos son de $C$. laevigatus. Sin embargo, el CV para longitud del culmo muestra en las tres especies tendencia a la homogeneidad ( CV $<20)$; mientras que la longitud del limbo manifiesta tendencia a la homogeneidad solamente en S. californicus; más no así en las otras dos especies. Los demás caracteres, carecen de importancia económica.

Igualmente, en la Tabla 2, se presenta la variabilidad estimada por especie; con homogeneidad en la mediana $(\mathrm{Mn}=1)$ en base a caracteres cualitativos; no así, en base a caracteres cuantitativos; con promedio más alto en $T$. dominguensis y el más bajo en $C$. laevigatus. La dispersión, en todos los casos, es amplia (CV>> 20).

El análisis de varianza, dentro y entre especies; indica que, estadísticamente $(\alpha=$ $0,05)$, no hay diferencias fenotípicas dentro de las especies; pero sí hay entre especies. El promedio de Typha dominguensis, aunque es mayor; estadísticamente (Prueba de Tukey), es igual al de Schoenoplectus californicus; y difieren con el valor medio de C. laevigatus.

Tabla 1. Ubicación taxonómica de las especies seleccionadas

\begin{tabular}{cccccc}
\hline División & Clase & Orden & Familia & Genero & Especie \\
\hline \multirow{2}{*}{ Magnoliophyta } & \multirow{2}{*}{ Liliopsida } & \multirow{2}{*}{ Poales } & Cyperaxease & Cyperus & C. laevigatus \\
& & & Syphaceae & $\begin{array}{c}\text { Schoplectus } \\
\text { Typha }\end{array}$ & $\begin{array}{c}\text { S. californicus } \\
\text { T. domingensis }\end{array}$
\end{tabular}


Tabla 2. Variabilidad intra e intrespecifica por OTUs y por carácter; más el ANVA dentro y entre especies

\begin{tabular}{|c|c|c|c|c|c|c|c|c|c|c|}
\hline \multirow{3}{*}{ Especies } & \multicolumn{7}{|c|}{ POR UNID ADES TAX ONÓMICAS OPERA TIVAS (OTUs) } & \multicolumn{3}{|c|}{ POR CARÁCTER } \\
\hline & \multirow{2}{*}{ Individuos- } & \multicolumn{3}{|c|}{ Cual itafivo (n=34) } & \multicolumn{3}{|c|}{ Cuartitativo(n=13) } & \multirow{2}{*}{ Carácter } & \multirow[t]{2}{*}{$\bar{x}$} & \multirow{2}{*}{$\mathrm{cV}$} \\
\hline & & Mn & $\mathbf{s}$ & $\mathrm{CV}$ & $\bar{x}$ & $\mathbf{s}$ & $\mathrm{cV}$ & & & \\
\hline \multirow{14}{*}{ 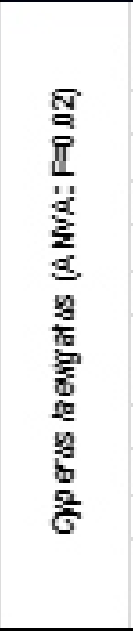 } & 1 & 1 & 0,44 & 51,08 & 13,16 & 30,05 & 228,27 & L oumo (cm) & 162,54 & 11,85 \\
\hline & 2 & 1 & 0,44 & 51,08 & 16,6 & 42 & 252,66 & D. almo (mm) & 3,62 & 24,06 \\
\hline & 3 & 1 & 0,44 & 51,08 & 19,7 & 48,7 & 247,26 & L. imbo (cm) & 22.92 & 38,74 \\
\hline & 4 & 1 & 0,44 & 51,08 & 18,5 & 42,8 & 230,83 & A imbo $(\sigma \mathrm{m})$ & 1,1769 & 26,45 \\
\hline & 5 & 1 & 0,44 & 51,08 & 18,5 & 49,4 & 267,81 & Lfam (mm) & 3.69 & 21,79 \\
\hline & 6 & 1 & 0,44 & 51,08 & 18,9 & 44,5 & 235,42 & L ane (mm) & 1,31 & 39,91 \\
\hline & 7 & 1 & 0,44 & 51,08 & 17,1 & 42,7 & 249,09 & A ant (mm) & 0,02 & 112,44 \\
\hline & 8 & 1 & 0,44 & 51,08 & 17,6 & 42,9 & 244,07 & Lestio (mm) & 3,19 & 31,02 \\
\hline & 9 & 1 & 0,44 & 51,08 & 16,1 & 41,7 & 258,56 & L estg (mm) & 0,01 & 36,05 \\
\hline & 10 & 1 & 0,44 & 51,08 & 16,6 & 43,4 & 261,66 & L infor (mm) & 10,31 & 23,56 \\
\hline & 11 & 1 & 0.44 & 51,08 & 18,9 & 51,4 & 272,29 & A reor (mm) & 8,23 & 21,13 \\
\hline & 12 & 1 & 0.44 & 51,08 & 18,1 & 44,6 & 245,49 & L espog (mm) & 7,31 & 14,12 \\
\hline & 13 & 1 & 0.44 & 51,08 & 18,3 & 48,8 & 267,39 & A espg (mm) & 3,69 & 17,07 \\
\hline & TOTAL & 1 & 0,43 & 50,38 & 17,54 & $2, \pi$ & 50,36 & \multicolumn{3}{|c|}{$n=13$ indviouos por caracter } \\
\hline \multirow{14}{*}{ 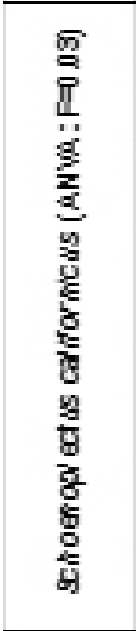 } & 1 & 1 & 0,35 & 38,82 & 49 & 67,1 & 167,76 & L oumo $(\mathrm{cm})$ & 289,38 & 8.3 \\
\hline & 2 & 1 & 0,35 & 34,82 & 39,3 & 74,4 & 189,25 & D aulmo (mm) & 15,24 & 28,99 \\
\hline & 3 & 1 & 0,35 & 3482 & 40,9 & 71,7 & 175,06 & L. imbo (cm) & 40,92 & 12,11 \\
\hline & 4 & 1 & 0,35 & 51,08 & 43,2 & 77,7 & 179,65 & A imbo (Gm) & 2,77 & 21,64 \\
\hline & 5 & 1 & 0,35 & 38,82 & 46,3 & 82,9 & 178,93 & Lfam (mm) & 0,11 & 20,54 \\
\hline & 6 & 1 & 0,35 & 3482 & 38,2 & 74,2 & 194,5 & L. ane (mm) & 0,02 & 86.93 \\
\hline & 7 & 1 & 0,35 & 3482 & 43,7 & 82,1 & 188,11 & A ant (mm) & 0,01 & 26,57 \\
\hline & 8 & 1 & 0,35 & 51,08 & 40,3 & 78,2 & 193,76 & L estio $(\mathrm{mm})$ & 1,79 & 43,87 \\
\hline & 9 & 1 & 0,35 & 38,82 & 51 & 83,3 & 163,13 & L estg (mm) & 0,82 & 11,37 \\
\hline & 10 & 1 & 0,35 & 34,82 & 39,9 & 75,5 & 188,99 & L infor (mm) & 7269 & 18.94 \\
\hline & 11 & 1 & 0,35 & 34,82 & 46,9 & 83,1 & 177,05 & A rifor (mm) & 53,85 & 50,54 \\
\hline & 12 & 1 & 0,35 & 51,08 & 46,8 & 91,3 & 194,78 & Lespg (mm) & 55,85 & 20,75 \\
\hline & 13 & 1 & 0,35 & 38,82 & 39,8 & 83,8 & 210,48 & A espo $(\mathrm{mm})$ & 23.08 & 19.82 \\
\hline & TOTAL & 1 & 0,34 & 3434 & 4281 & 7,31 & 178,25 & \multicolumn{3}{|c|}{$n=13$ indviouos por carácter } \\
\hline \multirow{17}{*}{ 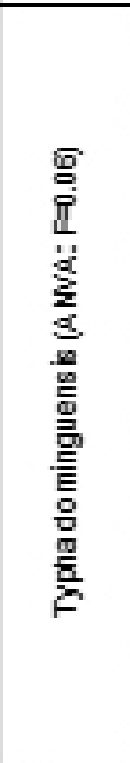 } & 1 & 1 & 0,39 & 37,7 & 53,2 & 103,5 & 194,8 & L oumo (cm) & 321.44 & 11,38 \\
\hline & 2 & 1 & 0,39 & 37,7 & 48,9 & 100,5 & 205,45 & Dalmo (mm) & 8,31 & 55,86 \\
\hline & 3 & 1 & 0,39 & 37,7 & 424 & 90,6 & 213,72 & L. Imbo (cm) & 175,6 & 249 \\
\hline & 4 & 1 & 0,39 & 37,7 & 49,7 & 124,4 & 209,88 & A ímbo $(\sigma m)$ & 2.22 & 88,19 \\
\hline & 5 & 1 & 0,39 & 37,7 & 62,8 & 111,1 & 176,97 & Lfam (mm) & 0,11 & 70,31 \\
\hline & 6 & 1 & 0,39 & 37,7 & 57,6 & 105,5 & 183,09 & L. ane (mm) & 0,03 & 5231 \\
\hline & 7 & 1 & 0,39 & 37,7 & 43,4 & 83,3 & 191,85 & $A$ ant $(\mathrm{mm})$ & 0,01 & 62,3 \\
\hline & 8 & 1 & 0,39 & 37,7 & 46,4 & 81,3 & 175,19 & L. estio (mm) & 0,31 & 72,69 \\
\hline & 9 & 1 & 0,39 & 37,7 & 48,1 & 125,9 & 220,47 & L esta (mm) & 0,02 & 56,37 \\
\hline & 10 & 1 & 0,39 & 37,7 & 41,7 & 79,1 & 189,59 & L infor (mm) & 44,5 & 46,32 \\
\hline & 11 & 1 & 0,39 & 37,7 & 43,1 & 83 & 192,71 & A rifor (mm) & 19,44 & 73,38 \\
\hline & 12 & 1 & 0,39 & 37,7 & 47,2 & 97 & 205,77 & L espg (mm) & 28,13 & 25,7 \\
\hline & 13 & 1 & 0,39 & 37,7 & 43,7 & 88,4 & 202,33 & A esogi(mm) & 36.56 & 15.31 \\
\hline & 14 & 1 & 0,39 & 37,7 & 54,7 & 101,1 & 184,75 & \multirow{4}{*}{\multicolumn{3}{|c|}{ 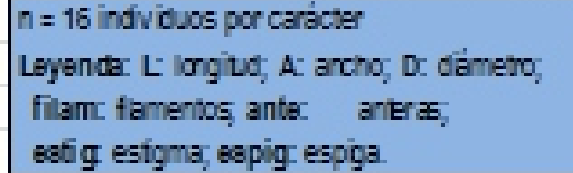 }} \\
\hline & 15 & 1 & 0,39 & 37,7 & 44,5 & 96,3 & 216,16 & & & \\
\hline & 16 & 1 & 0.39 & 37,7 & 5620 & 98,4 & 175,09 & & & \\
\hline & TOTAL & 1 & 0,38 & 37,18 & 4896 & 92,74 & 189,36 & & & \\
\hline
\end{tabular}




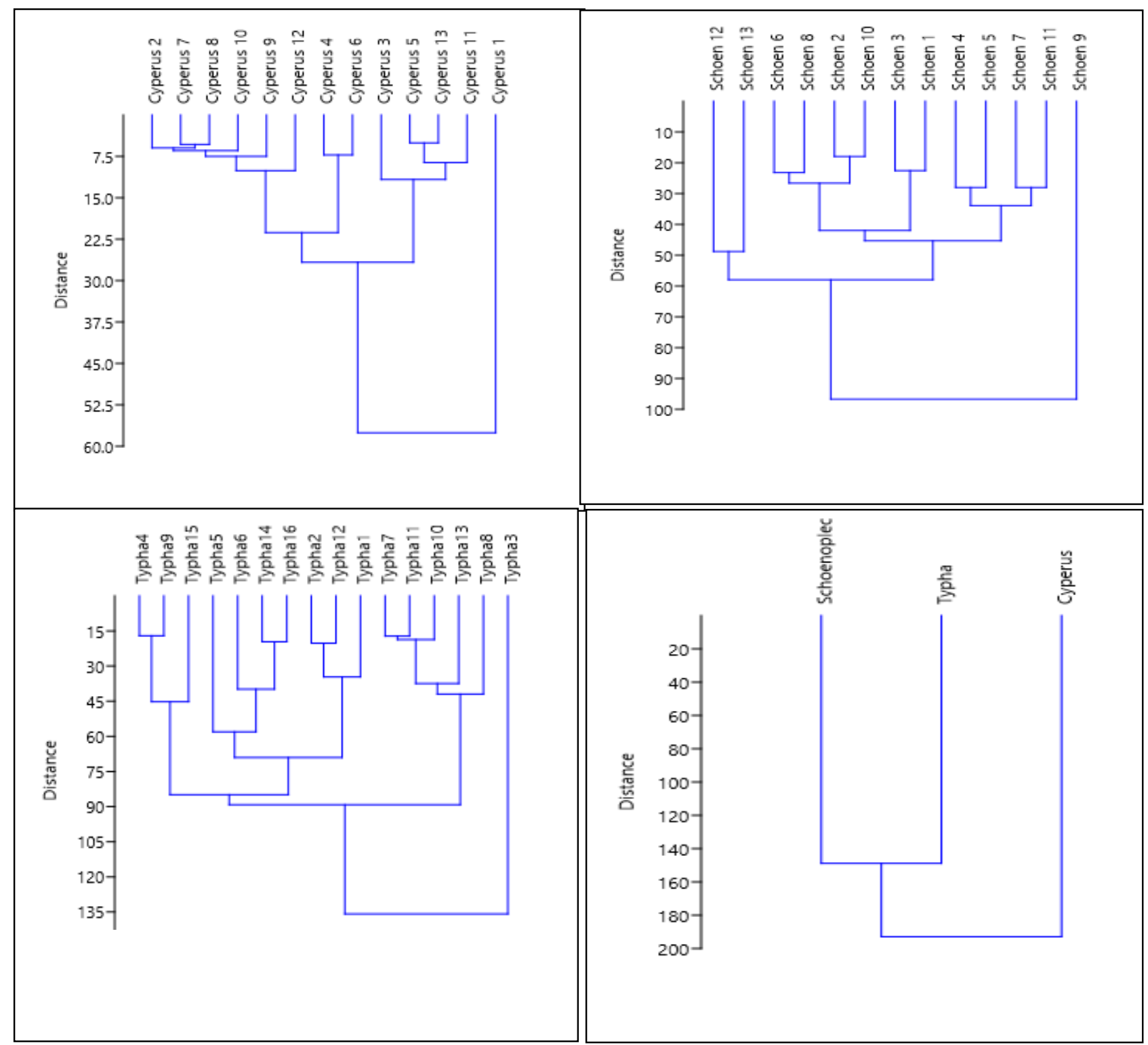

Figura 1. Fenogramas de la distancia fenética intra e interespecífica

En la Figura 1, se presentan los fenogramas de distancias fenéticas intraespecíficas ( $C$. laevigatus, S. californicus y T. dominguenis) e interespecíficas; obtenidos con el programa Past 3.0, mediante la técnica de agrupación de la media aritmética no ponderada (UPGMA).

El fenograma de $C$. laevigatus, muestra que la OTU 1 (Cyperus 1) es la más alejada; y los otros individuos se reúnen en dos grandes grupos y varios subgrupos. Un grupo lo conforman los individuos 3, 5, 11 y 13; constituyendo el otro grupo los restantes individuos; dentro del cual resalta el subgrupo formado por Cyperus 4 y Cyperus 6; y el subgrupo que reúne a los individuos $2,7,8,9$, 10 y 12 ; los cuales conforman más subgrupos.
En S. californicus, la OTU 9 (Schoen 9) es la más distante. Los demás individuos, se reúnen en tres grandes grupos y varios subgrupos. Un grupo está conformado por las OTUs 12 y 13; un segundo grupo lo constituyen dos subgrupos; donde las OTUs 4 y 5 conforman el subgrupo 1 ; y, las OTUs 7 y 11 representan al subgrupo 2 . En el tercer grupo las OTUs se reúnen en dos subgrupos; donde los ejemplares 1 y 3 constituyen el subgrupo 1; mientras que el subgrupo 2 está conformado por los ejemplares 6 y 8 (infragrupo 1) y las OTUs 2 y 10 (nfragrupo 2 ).

El fenograma de $T$. dominguensis, muestra que la OTU 3 (Typha 3) es la más alejada. Los 
demás individuos, se reúnen en tres grandes grupos y varios subgrupos. La heterogeneidad fenética intraespecífica de Typha dominguensis es mayor que las especies anteriores.

El fenograma de la distancia fenética interespecífica (C. laevigatus, S. californicus y $T$. angustifolia), muestra que $C$. laevigatus es el de menor parecido fenético (distancia mayor de 180); mientras que la distancia entre $S$. californicus y T. dominguensis se halla en el rango de 140 y 150.

\section{DISCUSIÓN}

Los ecosistemas de humedales son considerados entre los más productivos del mundo, lo cual favorece la reducción del proceso de eutroficación de los cuerpos de agua; además de proporcionar beneficios directos, a través del aprovechamiento de sus recursos bióticos; tanto en la alimentación como en cestería y textilería (Moschella, 2012).

Por ello, el poblador peruano desde épocas precolombinas hasta la actualidad, viene aprovechando recursos florísticos, propios de los humedales, para la confección de diversos artículos (sombreros, cestas, bolsas y sogas) y múltiples aplicaciones; tales como las islas flotantes de los uros en el lago Titicaca y los caballitos de totora en Huanchaco; todos ellos elaborados especialmente a base de Schoenoplectus californicus, Typha dominguensis y Cyperus laevigatus (Ministerio de Cultura, 2017).

Tal importancia, es reconocida por el Estado peruano, a través de la Resolución del Ministerio de Cultura (2017), que resuelve " Declarar Patrimonio Cultural de la Nación a los Conocimientos y prácticas relacionados a la cestería en junco y totora en las provincias de Huaura, Huaral y Barranca del departamento de Lima por constituir un arte tradicional ancestral basado en el uso milenario y sostenible del junco y la totora, en el que se expresan conocimientos técnicos y artísticos transmitidos de generación en generación, manteniendo continuidad y a la vez innovando en la producción" (Res. Viceministerial $\mathrm{N}^{\circ}$ 054-2015-VMPCIC, 2015).
En directa relación con los argumentos antes expuestos; es que, en el presente trabajo de investigación, se eligieron a las especies Schoenoplectus californicus, Typha dominguensis y Cyperus laevigatus por su importancia económica, histórica y cultural; al estar estrechamente relacionadas con el poblador peruano desde épocas precolombinas.

Por otro lado, tradicionalmente, con fines de selección para el mejoramiento genético respecto a los distintos caracteres de importancia económica, la diferenciación intraespecífica de los individuos se ha basado en su variabilidad fenotípica, por ser el resultado de la expresión de la diversidad genotípica (Amador Arranz, 2012). Además, los caracteres cuantitativos son mayormente poligénicos; presentando formas fenotípicas diferentes, que serían la expresión de la variabilidad intra e interespecífica (Castro Gómez et al. 2015).

Pero, la variabilidad fenotípica dentro y entre especies, es el resultado de la interacción genotipo - ambiente; de allí que organismos con idéntico genotipo, en condiciones ambientales heterogéneas, pueden presentar diferente fenotipo, y; ante homogeneidad de influencia ambiental, individuos con diferente genotipo, pueden presentan el mismo fenotipo para un determinado carácter (Amador Arranz, 2012).

El enmascaramiento del genotipo por influencia ambiental, es debido, principalmente, a la deficiente capacidad de expresión del genotipo; o como respuesta adaptativa de los organismos a la presión ambiental; por lo que, en estos casos, se recurre a métodos biotecnológicos genómicos y transcriptómicos, a fin de conocer la secuencia genética y su capacidad de expresión (Castro Gómez et al. 2015).

Tales razones, explicarían los resultados obtenidos en el presente trabajo; que, en general, muestran una baja variabilidad intra e interespecífica; lo que se observa más claramente en la distancia fenotípica estimada en los fenogramas respectivos; donde la especie $C$. laevigatus es el más alejado, al ser comparada con S. californicus y $T$. dominguensis. 
Las mismas razones explicarían la baja variabilidad detectada para caracteres cuantitativos; especialmente para longitud del culmo, que constituye el carácter de mayor importancia económica; y que se utiliza para elaborar diversos artículos. Por tanto, descartar si la poca variabilidad detectada es debida al genotipo o es causada por influencia ambiental, obliga aplicar métodos de secuenciación de ADN y de expresión génica, en un siguiente trabajo.

El análisis varianza y Prueba de Tukey aplicados para estimar diferencias fenotípicas dentro y entre especies, muestran la estrecha variabilidad intraespecífica expresada por la ausencia de diferencias significativas. Así mismo, tales análisis, muestran que fenotípicamente $C$. laevigatus es diferente a $S$. californicus y $T$. dominguensis; las mismas que son iguales entre sí; lo que se ratifica con el fenograma correspondiente (Figura 1).

\section{REFERENCIAS BIBLIOGRÁFICAS}

Amador Arranz, C. (2012). Estrategias de manejo poblacional para revertir introgresión de genes exógenos. Tesis para optar el grado de Doctor. Universidad Complutense de Madrid, Madrid.

Aponte, H., \& Cano, A. (2013). Estudio Florístico Comparativo de Seis Humedales de la Costa deLima (Perú): Actualización y Nuevos Retos para su Conservación. Revista Latinoamericana de Conservación, 3(2), 15-27.

Castro Gómez, J. C., Bombarely Gómez, A., Botella Mesa, M. Á., Marapara del Águila, J. L., Cobos Ruiz, M., \& Imán Correa, S. A. (2015). Análisis estructural y funcional del genoma de Myrciaria dubia "camu-camu" como base para su mejoramiento genético. Iquitos: Univ. Nac. de la Amazonía Peruana.

Crisci, J. V., \& Armengol, M. F. L. (1983). Introducción a la teoría y práctica de la taxonomía numérica (No. 57.06 CRI). Washington^ ${ }^{\wedge}$ D. CDC: OEA.

Dugan, P. J. (Ed.). (1992). Conservación de humedales: Un análisis de temas de actualidad y acciones necesarias. IUCN.
Huaringa Joaquín, A. W., Huanuqueño Coca, E., Camarena Mayta, F., \& Chura Chuquicaja, J. (2013). Manual de prácticas de fitomejoramiento general (No. F30. H87). Universidad Nacional Agraria La Molina, Lima (Peru).

Ministerio de Cultura. (20 de noviembre de 2017). Producción e Innovación Tecnológica de Textiles. Obtenido de c a $\mathrm{r}$ a $\mathrm{l}, \mathrm{g}$ o b . $\mathrm{p}$ e : http://www.caralperu.gob.pe/caralperu/c ivilizacion/civilizaciontextiles.html

Mitsch, W. J., \& Gosselink, J. G. (1993). Wetlands (Second edn).

Moschella Miloslavich, P. (2012). Variación y Protección de Humedales Costeros frente a Procesos de Urbanización: Casos de Ventanilla y Puerto Viejo. Lima: Pontificia Univ. Católica del Perú.

Núñez Colín, C. A., \& Escobedo López, D. (2011). Uso correcto del Análisis Cluster en la caracterización de Germoplasma Vegetal. Agronomía Mesoamericana, 22(2), 415-427.

Ostle B. (1981). Estadística Aplicada. México D.F.: LIMUSA.

Ramsar. (4 de Noviembre de 2016). Ramsar. $\mathrm{O} b \mathrm{te} \mathrm{n}$ i o d e http://www.ramsar.org/es/sitiospa\%C3\%ADses/sitios-ramsar-en-todoel-mundo.

Ministerio de Cultura (2015, Noviembre, 27). Resolución Viceministerial $N^{\circ} 054-2015-$ VMPCIC-MC. Declaran Patrimonio Cultural de la Nación a los Conocimientos y prácticas relacionados a la cestería en junco y totora en las provincias de Huaura, Huaral y Barranca del departamento de Lima. Diario Oficial El Peruano, 551448-551450.

\section{Correo electrónico:}

milabe48@hotmail.com

Revisión de pares:

Recibido:

Aceptado: 